\title{
Call for Papers: Progress Reports
}

Scholars are invited to write "progress reports" on topics of interest to the readers of Memory \& Cognition. We are seeking targeted assessments of extant phenomena (e.g., the generation effect; the mirror effect) and/or paradigms (e.g., DRM; process dissociation) - not literature reviews per se. These are meant to be progress, or accountability, reports - perhaps of the sort you might write for a granting agency to justify continued funding.

To accomplish the goal authors will need to follow a fixed structure, one designed to address accountability issues head-on. These articles may be controversial—for example, someone might encourage the field to stop doing research on $\mathrm{X}$ - and replies/commentaries will be encouraged. All submitted manuscripts will undergo the normal peer-review process and must adhere to current word limits $(8,000$ words). Here is the general organizational scheme:

1. Problem: Begin by placing the phenomenon you are discussing in some kind of functional context: We choose to study X, or adopt procedure X, because it is likely to help us understand "....." about memory, cognition, etc. This section should justify why the phenomenon or paradigm is interesting from a psychological standpoint.

2. Impact: Specifically address how $X$ has changed the way we think about memory and/or cognition. For instance, how would the field be different if $\mathrm{X}$ had never been discovered or developed? How has work on $\mathrm{X}$ changed the literature in a positive direction?

3. Trajectory: What do we know currently that we didn't know when X was first discovered or developed? Is the study of $\mathrm{X}$ near a point of diminishing returns? Are we making empirical progress, or simply tweaking parameters in response to previously tweaked parameters? How close has X brought us to solving the functional problem outlined in the first question?

4. Closure: What are the main unresolved issues-empirical and theoretical? Obviously, without a good answer to the first question - what cognitive problem is the study of X designed to solve? - it will be hard to gauge progress and understand what remains to be done.

5. Judgment: Summarize the arguments for and against continued work on X.

The field needs occasional "progress" or "accountability" reports, and Memory \& Cognition is a nice outlet. The field will appreciate pointed assessments by knowledgeable scholars about the tools and phenomena of our trade.

James S. Nairne Editor 